\title{
PIANO: A Web Server for Pseudouridine-Site ( $\Psi$ ) Identification and Functional Annotation
}

\author{
Bowen Song ${ }^{1 \dagger}$, Yujiao Tang ${ }^{1,2 \dagger}$, Zhen Wei ${ }^{1,3}$, Gang Liu ${ }^{4}$, Jionglong Su ${ }^{4}$, Jia Meng ${ }^{1,2}$ \\ and Kunqi Chen ${ }^{1,3 *}$
}

${ }^{1}$ Department of Biological Sciences, Xi'an Jiaotong-Liverpool University, Suzhou, China, ${ }^{2}$ Institute of Integrative Biology, University of Liverpool, Liverpool, United Kingdom, ${ }^{3}$ Institute of Ageing \& Chronic Disease, University of Liverpool, Liverpool, United Kingdom, ${ }^{4}$ Department of Mathematical Sciences, Xi'an Jiaotong-Liverpool University, Suzhou, China

OPEN ACCESS

Edited by:

Pavel V. Baranov,

University College Cork,

Ireland

Reviewed by:

Katsura Asano,

Kansas State University,

United States

Ivan V. Kulakovskiy,

Engelhardt Institute of Molecular

Biology (RAS), Russia

Claudio Lo Giudice,

National Research Council,

Italy

*Correspondence: Kunqi Chen

kunqi.chen@xitlu.edu.cn

${ }^{\text {t}}$ These authors have contributed equally to this work

Specialty section: This article was submitted to RNA, a section of the journal

Frontiers in Genetics

Received: 11 November 2019 Accepted: 27 January 2020

Published: 12 March 2020

Citation:

Song B, Tang Y, Wei Z, LiU G, SU J,

Meng J and Chen K (2020) PIANO: A Web Server for

Pseudouridine-Site ( $\Psi)$ Identification and

Functional Annotation.

Front. Genet. 11:88.

doi: 10.3389/fgene.2020.00088
Known as the "fifth RNA nucleotide", pseudouridine ( $\Psi$ or psi) is the first-discovered and most abundant RNA modification occurring at the Uridine site, and it plays a prominent role in a number of biological processes. Thousands of $\Psi$ sites have been identified within different biological contexts thanks to the advancement in high-throughput sequencing technology; nevertheless, the transcriptome-wide distribution, biomolecular functions, regulatory mechanisms, and disease relevance of pseudouridylation are largely elusive. We report here a web server-PIANO-for pseudouridine site $(\Psi)$ identification and functional annotation. PIANO was built upon a high-accuracy predictor that takes advantage of both conventional sequence features and 42 additional genomic features. When tested on six independent datasets generated from four independent $\Psi$-profiling technologies ( $\Psi$-seq, RBS-seq, Pseudo-seq, and CeU-seq) as benchmarks, PIANO achieved an average AUC of 0.955 and 0.838 under the full transcript and mature mRNA models, respectively, marking a substantial improvement in accuracy compared to the existing in silico $\Psi$-site prediction methods, i.e., PPUS (0.713 and 0.707), iRNA-PseU (0.713 and 0.712), and PseUI (0.634 and 0.652). Besides, PIANO web server systematically annotates the predicted $\Psi$ sites with post-transcriptional regulatory mechanisms (miRNA-targets, RBP-binding regions, and splicing sites) in its prediction report to help the users explore potential machinery of $\Psi$. Moreover, a concise query interface was also built for 4,303 known $\Psi$ sites, which is currently the largest collection of experimentally validated human $\Psi$ sites. The PIANO website is freely accessible at: http:// piano.rnamd.com.

Keywords: pseudouridine sites, genome-derived feature, RNA modification, Web-server, functional annotation

\section{INTRODUCTION}

Pseudouridine (5-ribosyluracil, $\Psi$, and psi) is the first-discovered (Cohn and Volkin, 1951) and most abundant RNA modification occurring at the Uridine site catalyzed by 13 pseudouridine synthase (PUS) (Chen and Patton, 2000; Zhao et al., 2004; McCleverty et al., 2007; Shaheen et al., 2016; Jacob et al., 2017). $\Psi$ is present in many classes of RNA within all organisms, such as 
messenger RNA (mRNA), transfer RNA (tRNA), small nucleolar RNA (snoRNA), small nuclear RNA (snRNA), and ribosomal RNA (rRNA) (Ge and Yu, 2013). $\Psi$ was termed as "the fifth nucleotide" with an estimated $\Psi / U$ ratio of 7-9\% (Jacob et al., 2017), and it is considered to be the most prevalent of the mRNA modifications (Meyer and Jaffrey, 2017). $\Psi$ plays a prominent role in many biological processes. The presence of $\Psi$ in tRNA and rRNA regulates the entry site binding process in ribosomal RNA (Jack et al., 2011) and RNA structure stabilization (Kierzek et al., 2014). A recent study also demonstrated that $\Psi$ is related to transcript stability (Schwartz et al., 2014), environmental signal response (Carlile et al., 2014), and genetic code switching in mRNA (Karijolich and Yu, 2011; Fernández et al., 2013). $\Psi$ deficiency may be associated with various diseases. It has been found that the dysregulation of $\Psi$ modification of mitochondrial tRNA acts as an etiology of mitochondrial myopathy and sideroblastic anemia (MLASA) (Bykhovskaya et al., 2004). Furthermore, mutations in pseudouridine are also involved in diseases like lung cancer and duykeratosis congenita (Mei et al., 2012).

Several high-throughput sequencing approaches have been developed for profiling the transcriptome-wide distribution of $\Psi$, including Pseudo-seq (Carlile et al., 2014), $\Psi$-seq (Schwartz et al., 2014), PSI-seq (Lovejoy et al., 2014), and CeU-seq (Li X,et al., 2015). These approaches all share the same principle, in which RNA is treated with the N-cyclohexyl-N'-(2-morpholinoethyl)carbodiimide-metho- $p$-toluenesulfonate (CMC) to leave a bulky group on $\Psi$ and stop reverse transcription. Since the bulky adduct on the $\Psi$ may reduce the sensitivity in the detection of $\Psi$, Vahid et al. recently developed a new approach, RBS-seq, which is based on a modification of RNA bisulfite sequencing and claims better sensitivity (Khoddami et al., 2019). Currently, the experiment-validated $\Psi$ sites in human, mouse, and a few other model organisms are available from RMBase database (Xuan et al., 2017), and the regulation pathways of $\Psi$ were more explicitly explained in MODOMICS database (Boccaletto et al., 2017).

Wet-lab approaches are surely effective for the study of transcriptome pseudouridylation with respect to a specific biological context; however, they are also laborious and offer only limited coverage, i.e., the reported RNA $\Psi$ sites by wet-lab experiments are still restricted to the transcripts more readily expressed under a specific cell/tissue condition. Alternatively, computational efforts may provide a more cost-effective avenue (Chen X, et al., 2017). To date, many computational efforts have been made to facilitate the study of RNA epigenetics (Boccaletto et al., 2017; Chen X, et al., 2017; Chen Z, et al., 2019; Xue et al., 2020; Liu et al., 2020) in terms of both experimental data collection and site prediction works. For predictors related to the identification of $\Psi$ RNA modification, PseUI (He et al., 2018), XG-PseU (Liu et al., 2019), and iRNA-PseU (Chen et al., 2016) allow for prediction of putative $\Psi$ sites from an RNA sequence, and PPUS (Li Y.H, et al., 2015) can predict the $\Psi$ sites regulated by a specific pseudouridine synthase. However, these three predictors are all based on sequence-derived features only without considering other genomic features (such as conservation, gene annotation, and miRNA binding) that may contribute to the prediction, and thus their performance is limited (Chen K, et al., 2019). Moreover, their prediction results are not functionally annotated with potential posttranscriptional regulation machineries that may explain the functional consequences of the predicted $\Psi$ sites.

We present here a web server-PIANO-for pseudouridine site identification and functional annotation. Inspired by the WHISTLE framework (Chen K, et al., 2019), PIANO took advantage of both the conventional sequence features and 42 additional genomic features. Using six independent datasets generated from four different technologies, we showed that PIANO adds a marked improvement to the accuracy of existing $\Psi$-site prediction. Moreover, the PIANO web server accepts both genomic location and RNA sequence format as input file when making predictions, and the putative $\Psi$ sites returned are also annotated with various post-transcriptional regulations, including miRNA-targets, RBPbinding regions, and splicing sites, to unveil potential functional mechanisms of $\Psi$. The PIANO website is freely accessible at: http:// piano.rnamd.com.

\section{MATERIALS AND METHODS}

\section{Training and Testing Data for $\Psi$-Site Prediction}

To construct the $\Psi$-site prediction model, we used the known human $\Psi$ sites detected from four different base-resolution $\Psi$ profiling techniques, including $\Psi$-Seq, RBS-Seq, CeU-Seq, and Pseudo-Seq (see Table 1). The $\Psi$ sites at base-resolution were directly downloaded from Gene Expression Omnibus (GEO).

In the beginning of the performance evaluation, dataset $\mathrm{H} 1$ (see Table 1) was used as the testing data, while dataset $\mathrm{H} 2-\mathrm{H} 4$ were used as for training. Specifically, the base-resolution $\Psi$ sites in training datasets were used as the positive training data. The negative sites used in model training were randomly selected from unmodified $U$ sites located on the same transcripts of positive sites (see Figure 1). To make the best use of the limited volume of positive data, we randomly selected 10 negative sites for

TABLE 1 | Base-resolution dataset used for $\Psi$-site prediction.

\begin{tabular}{|c|c|c|c|c|c|}
\hline Dataset & Cell line & Treatment & Technique & $\begin{array}{c}\text { Site } \\
\#\end{array}$ & Source \\
\hline $\mathrm{H} 1$ & HEK293 & & $\Psi$-Seq & 652 & $\begin{array}{l}\text { (Schwartz et al., } \\
\text { 2014) }\end{array}$ \\
\hline $\mathrm{H} 2$ & Hela & & RBS-Seq & 322 & $\begin{array}{l}\text { (Khoddami et al., } \\
\text { 2019) }\end{array}$ \\
\hline H3 & HEK293T & & CeU-Seq & 1555 & (Li X, et al., 2015) \\
\hline $\mathrm{H} 4$ & HEK293T & $\mathrm{H}_{2} \mathrm{O}_{2}$ & & 460 & \\
\hline H5 & HEK293T & $\begin{array}{l}\text { Heat Shock } \\
(\mathrm{HS})\end{array}$ & & 421 & \\
\hline $\mathrm{H} 6$ & Hela & & $\begin{array}{l}\text { Pseudo- } \\
\text { Seq }\end{array}$ & 156 & (Carlile et al., 2014) \\
\hline
\end{tabular}

The experimentally validated human $\Psi$ sites used in this project are also available from the PIANO website of this project (http://piano.rnamd.com), annotated with various posttranscriptional regulations. 


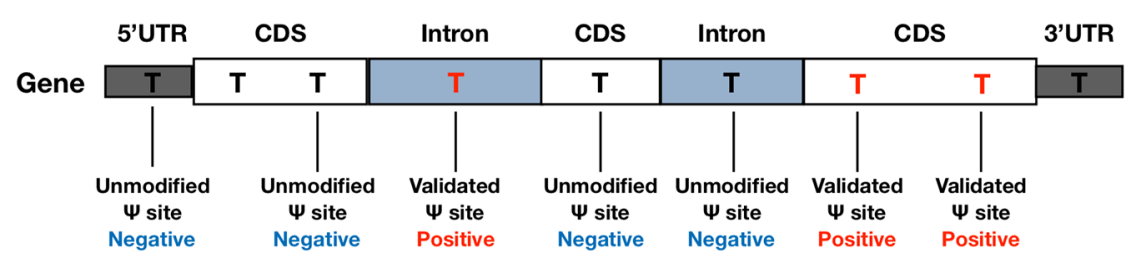

FIGURE 1 | Negative and Positive Data. Negative sites were randomly selected from un-modified U sites located on the same transcripts of the positive sites.

each of the positive sites. To balance the positive-to-negative ratio, the negative sites were then randomly split into 10 subsets, and 10 separate predictors were generated with a 1:1 positive-to-negative ratio. The negative sites of testing data were generated following the same procedure. Consequently, 10 separate predictors were generated, and their prediction results were averaged.

Following the experimental design of WHISTLE framework (Chen K, et al., 2019), we performed dataset level leave-one-out validation over the $\mathrm{H} 1-\mathrm{H} 5$ base-resolution datasets; four samples from $\mathrm{H} 1-\mathrm{H} 5$ were used as training, while the other was used for testing. Subsequently, the sites from the datasets $\mathrm{H} 1-\mathrm{H} 5$ (generated from $\Psi$-Seq, RBS-Seq, and CeU-Seq) were used to establish a predictor, whose performance was evaluated on the dataset H6, which was generated from an independent technology (Pseudo-Seq).

\section{Features Used for $\Psi$-Site Prediction Sequence-Derived Features}

The length of $41 \mathrm{bp}$ was widely used to extracted sequence information in many previous studies, which was determined as a suitable flanking window by relevant tests, i.e., iRNA-m7G (Liu et al., 2019), iRNA-2OM (Yang et al., 2018), and MethyRNA (Chen W, et al., 2017). Consequently, the sequence-derived information of 41 bp flanking window of $\Psi$ and non- $\Psi$ (U) sites as central was generated using the chemical properties of nucleotides, position-specific nucleotide propensity (PSNP), and cluster information.

In the first encoding method, the nucleotides are classified into three categories based on three distinct structural chemical properties. Ring structures of nucleotides are the first to be considered; here, adenosine and guanosine have two rings, while cytidine and uridine only have one ring. In addition, the guanosine and cytidine have stronger hydrogen bonding than adenosine and uridine. Furthermore, adenosine and cytidine can be classified as the amino group, while guanosine and uridine contain the keto group. Based on these chemical properties defined above, the $i$-th nucleotide from sequence $S$ may be encoded by a vector $S_{i}=\left(x_{i}, y_{i}, z_{i}\right)$ :

$$
x_{i}=\left\{\begin{array}{l}
1 \text { if } s_{i} \in\{\mathrm{A}, \mathrm{G}\} \\
0 \text { if } s_{i} \in\{\mathrm{C}, \mathrm{U}\}
\end{array}, y_{i}=\left\{\begin{array}{l}
1 \text { if } s_{i} \in\{\mathrm{A}, \mathrm{C}\} \\
0 \text { if } s_{i} \in\{\mathrm{G}, \mathrm{U}\}
\end{array}, z_{i}=\left\{\begin{array}{l}
1 \text { if } s_{i} \in\{\mathrm{A}, \mathrm{U}\} \\
0 \text { if } s_{i} \in\{\mathrm{C}, \mathrm{G}\}
\end{array}\right.\right.\right.
$$

Thus, the $A, C, G$, and $U$ may be encoded as a vector $(1,1,1)$, $(0,1,0),(1,0,0)$, and $(0,0,1)$, respectively.

The position-specific nucleotide propensity (PSNP) stands for the differences of the frequency of nucleotides calculated in specific locations between RNA sequences of positive and negative data. The frequency of occurrence of $A, U, G$, and $C$ in the $i$-position were calculated for both positive and negative data, respectively, to obtain two matrices with $4 \times 41$ dimension as $Z_{\text {plus }}$ and $Z_{\text {minus }}$, where $Z_{\text {plus }}$ was extracted from sequence of all positive data, and $Z_{\text {minus }}$ was extracted from sequence of all negative data. The position-specific nucleotide propensity (PSNP) matrices was defined as $\mathrm{Z}_{\mathrm{PSNP}}$ :

$$
Z_{\mathrm{PSNP}}=Z_{\text {plus }}=Z_{\text {minus }}
$$

For the cluster information, the average relative position of the closest $k(k=1,2$ and 3$)$ nucleotide to center $\Psi /$ non- $\Psi$ was calculated for each nucleic acid (A, G, C, and U). The $k$ was considered as 1 to 3 . Using sequence 'AGCUAGCCAUC CUACGGUACAGCAU' as an example, the center $U$ is at the ninth positive. For encoding the cluster information of adenine, the average relative position of the closest $1(\mathrm{k}=1)$ adenine to center $U$ is $1(1 / 1)$; when $k$ equals to 2 , the relative position of the second closest adenine to center $U$ is 4 , and, therefore, the average relative position of the closest $2(\mathrm{k}=2)$ adenine to center $\mathrm{U}$ is $2.5(5 / 2)$ and $3.7(11 / 3)$ when $\mathrm{k}$ equals to 3 . Similarly, the cluster information of guanosine in this example sequence is $3(3 / 1), 3.5(7 / 2)$, and $4.7(14 / 3)$ when k equals to 1,2 , and 3 , respectively.

The sequence-derived encoding methods employed by the three previously published predictors were used to reproduce the PPUS, iRNA-PseU, and PseUI with the same training data of PIANO, respectively, and their performances were compared with PIANO using independent datasets.

\section{Genome-Derived Features}

In the original WHISTE approach, 35 additional genomic features that might contribute to the prediction of $\mathrm{m}^{6} \mathrm{~A}$ RNA methylation sites were considered (Chen K, et al., 2019). In PIANO, seven new genomic features were added to the prediction model, the details of the 42 genomic features considered in the prediction were summarized in Supplementary Table S1. Specifically, genomic Features 116 are dummy variable features indicating whether the uridine sites shall fall within the transcript regions that satisfy certain topological properties. All the features in this category are generated by the GenomicFeatures R/Bioconductor package using the transcript annotations hg19 TxDb package (Lawrence et al., 2013). To remove the ambiguity caused by transcript isoforms, only the primary (longest) transcripts of 
each gene were kept for the extraction of the transcript subregions. The longest transcript isoform was used to unambiguously assign m6A peak regions to mRNAs (Ke et al., 2017) and contributed to a better performance in accuracy compared with using the average value of multiple transcripts. Genomic Features 17-20 are real valued features defining the relative position of the transcript regions (3'UTR, 5'UTR, CDS, and whole transcript), i.e., the distance from the adenine to the 5 ' end divided by the width of the region. The values are also set to zero for sites that do not belong to the region. Genomic features 21-25 represent the length of the transcript region containing the modification site. The values are also set to zero for sites that do not belong to the region. Features 26-27 captured the distance from the adenine sites to the 5 'end or 3 'end of the splicing junctions. Additionally, the distance to the nearest neighboring $\psi$ sites in the training data is generated to measure the clustering effect of the $\psi$ RNA modification sites. Evolutionary conservation score of the uridine sites and its flanking regions are measured by PhastCons (Siepel et al., 2005) score, and the fitness consequence (Gulko et al., 2015) scores were presented in features 28-31. To consider the RNA secondary structures around the uridine site, the RNA secondary structures are predicted using RNAfold from the Vienna RNA package (Lorenz et al., 2011) and presented in features 32-33. Genomic properties of transcripts containing the $\Psi$ sites were presented in features 34-38. Finally, features 39-42 represent omics information, such as microRNA target sites (Chou et al., 2017) and HNRNPC binding sites (2012).

\section{Machine Learning Approach Used for $\Psi$-Site Prediction}

As a high-efficiency machine learning algorithm in computational biology, the SVM (Support Vector Machine) has been widely applied in microRNA target prediction (Liu et al., 2010), protein phosphorylation prediction (Wong et al., 2007), and $\mathrm{m}^{6} \mathrm{~A}$ RNA methylation site prediction (Chen W, et al., 2017). In this project, the $\mathrm{R}$ language interface of LIBSVM (Chang and Lin, 2011) was used to build our model with the radial basis function as kernel, and the other parameters were set at the default.

\section{Performance Evaluation of $\Psi$-Site Prediction}

To evaluate the performance of PIANO, a 5-fold cross-validation was employed on training datasets using the SVM classifier, and the independent testing dataset was used to measure the final performance of PIANO. There is no overlap between the training sites and testing sites, as only the $\Psi$ sites not previously used as training data were considered during performance evaluation; the performance evaluation result should thus directly reflect the capability of the algorithm to identify previously unknown $\Psi$ sites. To evaluate the performance, the ROC (receiver operating characteristic) curve (sensitivity against 1-specificity) was used, and the area under ROC curve (AUROC) was calculated as the main performance evaluation metric.

\section{Estimate the Probability of $\Psi$}

The likelihood ratio (LR) of a $\Psi$ site is calculated to estimate the probability of $\Psi$ RNA methylation:

$$
L R=\frac{P(\text { observation } \mid \Psi)}{P(\text { observation } \mid \mathrm{U})}
$$

In the PIANO web server, a site was predicted to be a putative $\Psi$ site if its predictive value was above 0.5 with a minimum LR value of 1 . A site with a larger $L R$ value suggests that it is more likely to be a $\Psi$ site. The machine learning classifiers usually obtain the lowest empirical rate with the value of 0.5 as cutoff. The statistical significance of LR is assessed by an upper bound of the p-value, indicating how extreme the observed LR is among all the transcriptome $U$ sites. It is calculated from the relative ranking of the putative $\Psi$ sites among all the transcriptome $U$ sites, i.e., if only $0.1 \%$ of $U$ sites have a LR score larger than a specific $U$ site, then the upper bound of the $p$-value of this site is 0.001 . In the report of PIANO web server, a putative $\Psi$ site is considered to be of high confidence if its LR within the top $0.5 \%$ of all transcriptome Us (corresponding to an upper bound of the p-value $<0.005)$ of all the transcriptome $U$ sites, followed by medium confidence $(0.005<$ upper bound of the p-value $\leq 0.05)$ and low confidence ( $\mathrm{p}$-value $>0.05$ ).

\section{Functional Annotation of Putative $\Psi$ Site}

The gene symbol, Ensembl gene ID, gene region, and gene type for each putative $\Psi$ site were annotated using ANNOVAR package (Wang et al., 2010). Furthermore, we annotated the putative $\Psi$ sites with three kinds of post-transcriptional regulation, including RNA-binding proteins (RBPs) regions, miRNA-RNA targets, and splicing sites. We first found the intersection between the computational predicted $\Psi$ sites and POSTAR2-derived RBP binding regions (Zhu et al., 2018). For miRNA targets, we obtained the information from miRanda (Agarwal et al., 2015) and starBase2 (Li et al., 2013), and we found the $\Psi$ sites within the miRNA targets regions to explore the potential influence of $\Psi$ on miRNA-target interactions. Finally, we obtained the Canonical splice sites (GT-AG) from UCSC (Lawrence et al., 2013) annotations, 100 bp upstream region from 5' splicing sites and $100 \mathrm{bp}$ downstream region from 3 ' splicing sites were extracted for the subsequent analysis of $\Psi$ sites on splicing sites. The detailed information of the posttranscriptional regulation association analysis can be found in Supplementary Table S2.

\section{RESULTS}

Although the genome-derived features alone are already very effective for predicting $\Psi$ sites, the best performance was achieved when the sequence features and genomic features were combined. Consequently, our PIANO predictor was established based on both the genome-derived features and sequence-derived features. When designing the encoding methods for sequence features used for the PIANO approach, the chemical properties of nucleotides, position-specific nucleotide propensity (PSNP), and 
cluster information were considered. We found that this combination (sequence and genomic features) achieved the best performance in accuracy compared with combining genomederived features with other basic sequence encoding methods (i.e., one-hot encoding method).

The performance of the predictor was evaluated under two modes. For the full transcript mode, the positive and negative $\Psi$ sites located in both exonic and intronic regions are all considered to construct the predictor. In the mature mRNA mode, only positive and negative $\Psi$ sites located on mature mRNA transcripts are considered; this is because existing experimental datasets overwhelmingly relied on polyA selection in RNA-seq library preparation, and intronic $\Psi$ sites are likely to be underrepresented in the data, which may lead to an over-estimation of accuracy under the full transcript mode.

To avoid potential over-fitting and to identify the most significant subset of genomic features, feature selection was implemented; the datasets $\mathrm{H} 2-\mathrm{H} 5$ were used as training data, while dataset $\mathrm{H} 1$ was used for the independent testing data. The relative importance of each genome-derived feature were measured by the Perturb method (Gevrey et al., 2003). According to the rank of importance, the top $\mathrm{N}$ most important features were reserved in the prediction and were evaluated with a 5 -fold cross-validation. For the predictor under full transcript model, the top 17 genomic features led to the best predictor performance, with fitCons scores, exons containing stop codons, and number of exons as the top three most important genomic features for prediction. Similarly, the top 20 genome-derived features were selected under the mature mRNA model. The length of the mature transcript plays the most important role under this model, and the exons containing stop codons and an miRNA target won the second and third significance. Consequently, to obtain the most robust performance, only the top 17 and 20 genomic features were used under full transcript model and mature mRNA model for $\Psi$ site prediction, respectively. Please see Supplementary Figure S1 for more details.

We showed that the newly developed method PIANO substantially outperformed competing approaches on crossvalidation (Supplementary Table S3) when tested on independent datasets (Supplementary Table S3) or benchmarked by an independent technique (Supplementary Table S4). To sum up, by testing independent datasets generated from four different $\Psi$ profiling technologies ( $\Psi$-seq, RBS-seq, Pseudo-seq, and CeU-seq), the newly developed method PIANO achieved an average AUC of 0.955 and 0.838 under full transcript and mature mRNA modes, respectively (see Table 2), representing a marked improvement compared to PPUS (0.713 and 0.707), iRNA-PseU (0.713 and 0.712), and PseUI (0.634 and 0.652).

The performance of the purposed predictor was further evaluated by separating the training and testing datasets between the cell type in which datasets $\mathrm{H} 3-\mathrm{H} 5$ generated from HEK293T were used for training, while datasets $\mathrm{H} 2$ and $\mathrm{H} 6$ from Hela were used for independent testing. Consistent with previous validation results, our method PIANO achieved a marked
TABLE 2 | Performance evaluation of $\Psi$-site predictors.

\begin{tabular}{|c|c|c|c|c|c|c|}
\hline \multirow[t]{2}{*}{ Mode } & \multirow[t]{2}{*}{ Method } & \multicolumn{4}{|c|}{ Benchmarking data (AUC) } & \multirow{2}{*}{$\begin{array}{c}\text { Average } \\
\text { AUC }\end{array}$} \\
\hline & & $\begin{array}{l}\Psi- \\
\text { Seq }\end{array}$ & $\begin{array}{l}\text { RBS- } \\
\text { Seq }\end{array}$ & $\begin{array}{l}\text { CeU- } \\
\text { Seq }\end{array}$ & $\begin{array}{l}\text { Pseudo- } \\
\text { Seq }\end{array}$ & \\
\hline \multirow{4}{*}{$\begin{array}{l}\text { Full } \\
\text { transcript }\end{array}$} & PIANO & 0.957 & 0.978 & 0.914 & 0.972 & 0.955 \\
\hline & $\begin{array}{l}\text { iRNA- } \\
\text { PseU }\end{array}$ & 0.679 & 0.727 & 0.721 & 0.708 & 0.713 \\
\hline & PPUS & 0.700 & 0.721 & 0.724 & 0.705 & 0.713 \\
\hline & PseUl & 0.631 & 0.710 & 0.610 & 0.585 & 0.634 \\
\hline \multirow{4}{*}{$\begin{array}{l}\text { Mature } \\
\text { mRNA }\end{array}$} & PIANO & 0.859 & 0.770 & 0.864 & 0.857 & 0.838 \\
\hline & $\begin{array}{l}\text { iRNA- } \\
\text { PseU }\end{array}$ & 0.753 & 0.582 & 0.760 & 0.751 & 0.712 \\
\hline & PPUS & 0.749 & 0.575 & 0.757 & 0.748 & 0.707 \\
\hline & PseUl & 0.666 & 0.651 & 0.652 & 0.639 & 0.652 \\
\hline
\end{tabular}

The table presents the performance of different $\Psi$ site predictors achieved on independent human datasets with different technologies as a benchmark, and it is summarized from Supplementary Table S3 and S4. Only the $\Psi$ sites not previously used as training data were considered during performance evaluation, so the training sites and testing sites did not overlap. Because existing datasets overwhelmingly relied on polyA selection in RNA library preparation and intronic $\Psi$ sites are likely to be underrepresented in the data, the performances were evaluated under two modes: full transcript and mature mRNA modes. In the mature mRNA mode, only positive and negative $\Psi$ sites located on mature mRNA transcripts are considered, as previously described (Chen K,et al., 2019). Our new approach PIANO substantially outperformed competing approaches in accuracy.

improvement in prediction accuracy compared with existing predictors, using the AUROC (area under ROC curve) and AUPRC (area under precision-recall curve) as an evaluation metric, when tested on independent dataset with a 1:1 positive to negative ratio (Supplementary Table S5) and 1:10 positive to negative ratio (Supplementary Table S6), respectively, suggesting the reliability of our newly proposed approach. Besides, the comparison between different algorithms indicated that SVM (Support Vector Machine) was a quite effective machine learning approach and achieved the best performance in our study (Supplementary Table S5). In addition, to further evaluate different approaches, we also considered the prediction of PUS-specific $\Psi$ sites. In this experiment, TruB1, PSU7, and TruB2 were considered, and the goal was to predict their specific substrates (Safra et al., 2017). Consistent with previous results in $\Psi$-site prediction, the PIANO method again substantially outperformed competing approaches under both the full transcript and mature mRNA model (Table 3), suggesting the effectiveness of the approach.

\section{Construction of the PIANO Website}

A website PIANO, which stands for pseudouridine site identification and functional annotation, was built for the convenience of academic users. Hyper Text Markup Language

TABLE 3 | PUS-specific substrate prediction.

\begin{tabular}{|c|c|c|c|c|c|c|}
\hline \multirow[t]{2}{*}{ Method } & \multicolumn{3}{|c|}{ Full transcript model } & \multicolumn{3}{|c|}{ Mature mRNA model } \\
\hline & TruB2 & PSU7 & TruB1 & TruB2 & PSU7 & TruB1 \\
\hline PIANO & 0.981 & 0.966 & 0.973 & 0.837 & 0.960 & 0.910 \\
\hline iRNA-PseU & 0.812 & 0.829 & 0.838 & 0.719 & 0.812 & 0.731 \\
\hline PPUS & 0.806 & 0.824 & 0.824 & 0.733 & 0.816 & 0.739 \\
\hline PseUl & 0.853 & 0.870 & 0.840 & 0.805 & 0.861 & 0.786 \\
\hline
\end{tabular}


(HTML), Cascading Style Sheets (CSS), and Hypertext Preprocessor (PHP) were used to construct the PIANO web interface. This included a database containing 4,303 experimentally validated $\Psi$ sites reported from four different high-throughput $\Psi$ profiling techniques, which is so far the most complete collection of $\Psi$ in humans. Among those experimentally validated $\Psi$ sites, we found $\Psi$ was distributed most often along coding DNA sequence and 3'UTR, but it was relatively rare in 5'UTR (Supplementary Figure S2). Secondly, a web server for putative $\Psi$-site identification from the userdefined genomic ranges or provided FASTA sequences (detailed in Figure 2) was used. The help document of the PIANO web server is provided in the Supplementary Materials. Both experimentally validated $\Psi$ sites and the predicted putative $\Psi$ sites are functionally annotated with various posttranscriptional regulations to unveil potential functional mechanism concerning $\Psi$. The data and prediction results may be conveniently downloaded and visualized with web browser. The PIANO website is freely accessible from: http://piano. rnamd.com.

\section{CONCLUSION}

With recent advancements that unveiled various biomolecular functions of $\Psi$ under different biological contexts, $\Psi$ starts to capture broader interests of the scientific community (Schwartz et al., 2014; Carlile et al., 2014; Li X, et al., 2015; Karijolich et al., 2015; Dominissini et al., 2016; Penzo et al., 2017; Guzzi et al., 2018; Adachi et al., 2018; Shaheen et al., 2019). To date, a number of high-throughput approaches have been developed for profiling the transcriptome-wide distribution of $\Psi$ (Adachi et al., 2019), including Pseudo-seq (Carlile et al., 2014), $\Psi$-seq (Schwartz et al., 2014), PSI-seq (Lovejoy et al., 2014), CeU-seq (Li X, et al., 2015), and RBS-seq (Khoddami et al., 2019). These technologies all reported the widespread occurrence of $\Psi$ on mRNA and lncRNA in human cells. Four $\Psi$ site predictors have been built, including PseUI (He et al., 2018), XG-PseU (Liu et al., 2019), iRNA-PseU (Chen et al., 2016), and PPUS (Li Y.H, et al., 2015); however, all of them are based on sequence-derived features only without considering other genomic features that may contribute to the prediction and thus limited their performance.

Here, by integrating 42 genomic features together with conventional sequence-derived features, we have developed the (so far) most accurate $\Psi$-site predictor. Our new method (PIANO) substantially outperformed competing approaches when using four different $\Psi$ profiling protocols as the benchmarks (with 0.24 and 0.12 improvement in terms of AUC under full transcript and mature mRNA modes, respectively) and supports functional annotation for the putative $\Psi$ sites. A web site-PIANO-was also developed, including (1) a database hosting currently the largest collection of 4,303 experimentally validated human $\Psi$ sites; and (2) a web

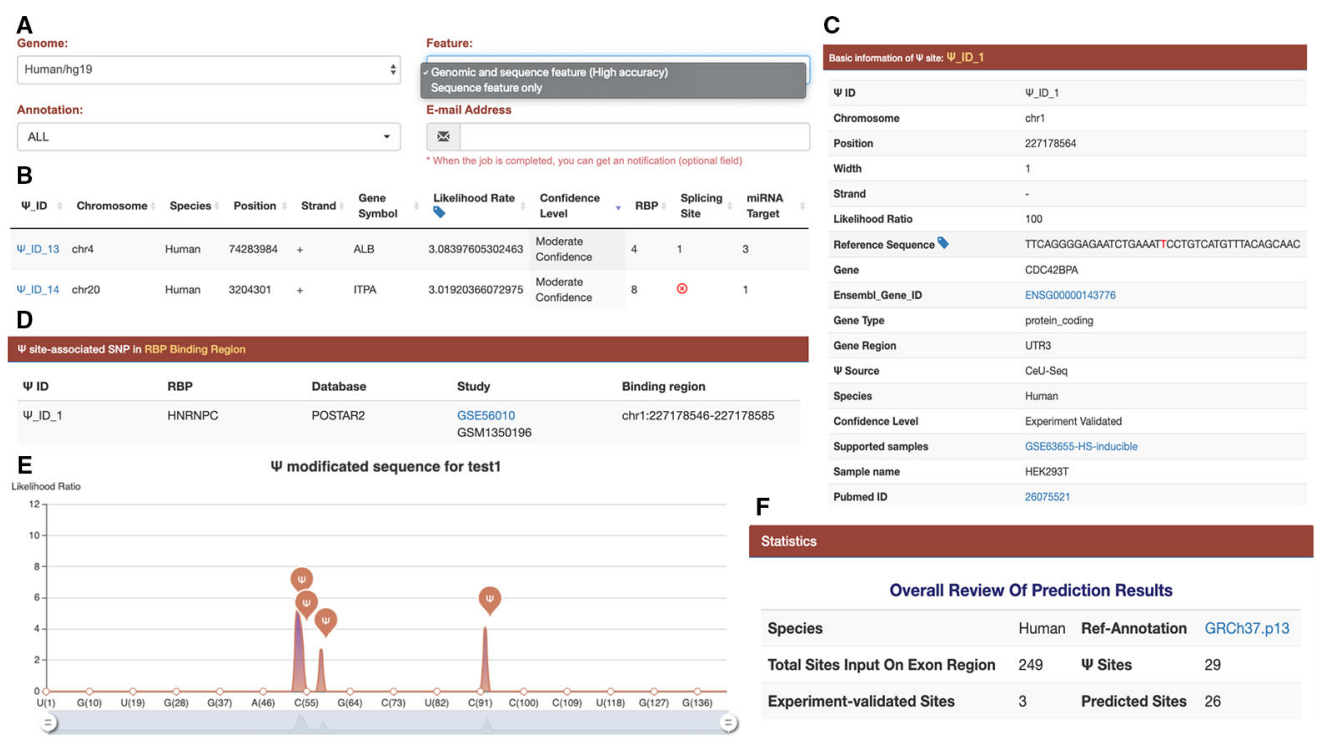

FIGURE 2 | Interface and output of the PIANO web server for $\Psi$-site prediction and functional annotation. (A) When predicting human $\Psi$ sites, the PIANO web server supports two types of input: the genomic ranges of human genome assembly and the FASTA sequences. As the prediction process may take quite some time, it is highly recommended that the user should provide an email address, where an email notification will be sent when the job is finished. (B) The basic information of each putative $\Psi$ site, such as gene symbol, likelihood ratio, confidence level, and the number of related post-transcriptions associated with the putative site. (C) The source and detailed information of each putative $\Psi$ site. If the input file contains any experimental validated $\Psi$ sites collected in PIANO, the sites will be annotated with additional information. (D) The details of the site-relevant RBP information. (E) A graph to visualize the position of predicted $\Psi$ sites on a userprovided FASTA sequence. (F) An overall review of the prediction result. 
server enabling the prediction of novel $\Psi$ sites from given genomic ranges or FASTA sequences. Users may query and download their predicted results with clear and simple instructions (see Supplementary Materials). The scripts used to generate genomic and sequence features considered in PIANO's framework, the training and testing data, and datasets related to the construction of the PIANO database were provided in the download page of PIANO website. In conclusion, our work will serve as a useful resource for researchers who are interested in $\Psi$ and its role concerning various post-transcriptional regulations.

Nevertheless, it is worth noting that there exist significant discrepancies in the $\Psi$ sites reported by different technologies (Zaringhalam and Papavasiliou, 2016; Adachi et al., 2018). Although the discrepancy may be due to the context-specificity of pseudouridylation and technology preferences, our PIANO predictor achieved reasonable consensus with all the four highthroughput profiling $\Psi$ techniques; $\Psi$ is, however, considered as the most prevalent mRNA modifications (Meyer and Jaffrey, 2017) with an estimated $\Psi / U$ ratio of 7-9\% (Jacob et al., 2017). Currently, only a small number of $\Psi$ sites have been reported; we are therefore not able to calculate a reasonable number for the real-life estimate of class imbalance. This may due to the limited detection power of existing experimental approaches. With an estimated real-life $\Psi / U$ ratio as $8 \%$, we can expect at least 10 times the number of negative sites. Under this assumption, we tested the stability of our method by assigning $1: 10$ and 1:1 positive-to-negative ratio for the training and testing data. The result showed that the performance generated by the 1:10 class were more stable than the 1:1 class (Supplementary Figure S3). We further calculated the value of FDR, FPR, and TPR in this setting, using different LRs as cutoff (Supplementary Table S7). To sum up, we cannot rule out the possibility of experimental bias, and the training data (gold standard data) may be further optimized in the future as more experimental evidence is

\section{REFERENCES}

(2012). An integrated encyclopedia of DNA elements in the human genome. Nature 489 (7414), 57-74.

Adachi, H., De Zoysa, M. D., and Yu, Y.-T. (2018). Post-transcriptional pseudouridylation in mRNA as well as in some major types of noncoding RNAs. Biochim. Biophys. Acta (BBA)-Gene Regul. Mech. 1862, 230-239.

Adachi, H., DeZoysa, M. D., and Yu, Y.-T. (2019). "Detection and Quantification of Pseudouridine in RNA," in Epitranscriptomics (Springer), 219-235.

Agarwal, V., Bell, G. W., Nam, J. -W., and Bartel, D. P. (2015). Predicting effective microRNA target sites in mammalian mRNAs. eLife 4, e05005.

Boccaletto, P., Machnicka, M. A., Purta, E., Piątkowski, P., Bagiński, B., Wirecki, T. K., et al. (2017). MODOMICS: a database of RNA modification pathways. 2017 update. Nucleic Acids Res. 46, D303-D307.

Bykhovskaya, Y., Casas, K., Mengesha, E., Inbal, A., and Fischel-Ghodsian, N. (2004). Missense mutation in pseudouridine synthase 1 (PUS1) causes mitochondrial myopathy and sideroblastic anemia (MLASA). Am. J. Hum. Genet. 74 (6), 1303-1308. doi: 10.1086/421530

Carlile, T. M., Rojas-Duran, M. F., Zinshteyn, B., Shin, H., Bartoli, K. M., and Gilbert, W. V. (2014). Pseudouridine profiling reveals regulated mRNA pseudouridylation in yeast and human cells. Nature 515 (7525), 143-146.

Chang, C.-C., and Lin, C.-J. (2011). LIBSVM: a library for support vector machines. ACM Trans. Intell. Syst. Technol. 2 (3), 1-27. doi: 10.1145/1961189.1961199 accumulated. To make the PIANO method more practically useful, the predictor should be used by combining with other experimental evidence and knowledge, e.g., the Us within a binding site of PUS. The performance of PIANO method is much better than all existing approaches, and it can provide the most reliable putative $\Psi$ sites for users.

\section{DATA AVAILABILITY STATEMENT}

Publicly available datasets were analyzed in this study. This data can be found here: GSE60047, GSE58200, GSE63655, GSE90963.

\section{AUTHOR CONTRIBUTIONS}

$\mathrm{KC}, \mathrm{JM}, \mathrm{GL}$, and JS initialized the project. KC and BS designed the research plan. ZW constructed the genomic features considered in human $\Psi$ site prediction. BS performed the development of the $\Psi$ site web server. YT and BS built the website. BS and KC drafted the manuscript. All authors read, critically revised, and approved the final manuscript.

\section{FUNDING}

This work has been supported by the National Natural Science Foundation of China [31671373]; XJTLU Key Program Special Fund [KSF-T-01].

\section{SUPPLEMENTARY MATERIAL}

The Supplementary Material for this article can be found online at: https://www.frontiersin.org/articles/10.3389/fgene.2020. 00088/full\#supplementary-material

Chen, J., and Patton, J. R. (2000). Pseudouridine synthase 3 from mouse modifies the anticodon loop of tRNA. Biochemistry 39 (41), 12723-12730. doi: 10.1021/bi001109m

Chen, W., Tang, H., Ye, J., Lin, H., and Chou, K. C.. (2016). iRNA-PseU: Identifying RNA pseudouridine sites. Mol. Ther. Nucleic Acids 5, e332.

Chen, X., Sun, Y. Z., Liu, H., Zhang, L., Li, J. Q., and Meng, J. (2017). RNA methylation and diseases: experimental results, databases, Web servers and computational models. Brief Bioinform. 20, 896-917.

Chen, W., Tang, H., and Lin, H. (2017). MethyRNA: a web server for identification of N6-methyladenosine sites. J. Biomol. Struct. Dynamics 35 (3), 683-687. doi: 10.1080/07391102.2016.1157761

Chen, Z., Zhao, P., Li, F., Wang, Y., Smith, A. I., Webb, G. I., Akutsu, T., et al. (2019). Comprehensive review and assessment of computational methods for predicting RNA post-transcriptional modification sites from RNA sequences. Briefings Bioinf. bbz112 doi: 10.1093/bib/bbz112

Chen, K., Wu, Q., Zhang, Z., Wei, R., Rong, Z., Lu, J., Meng, J. P., et al. (2019). WHISTLE: a high-accuracy map of the human N6-methyladenosine (m6A) epitranscriptome predicted using a machine learning approach. Nucleic Acids Res. 47, e41 doi: 10.1093/nar/gkz074

Chou, C. -H., Shrestha, S., Yang, C. -D., Chang, N. -W., Lin, Y. -L., Liao, K.-W., et al. (2017). miRTarBase update 2018: a resource for experimentally validated microRNA-target interactions. Nucleic Acids Res. 46 (D1), D296-D302.

Cohn, W. E., and Volkin, E. (1951). Nucleoside-5'-Phosphates from Ribonucleic Acid. Nature 167 (4247), 483-484. 
Dominissini, D., Nachtergaele, S., Moshitch-Moshkovitz, S., Peer, E., Kol, N., BenHaim, M. S., et al. (2016). The dynamic N(1)-methyladenosine methylome in eukaryotic messenger RNA. Nature 530 (7591), 441-446.

Fernández, I. S., Ng, C. L., Kelley, A. C., Wu, G., Yu, Y. -T., and Ramakrishnan, V. (2013). Unusual base pairing during the decoding of a stop codon by the ribosome. Nature 500 (7460), 107.

Ge, J., and Yu, Y. T. (2013). RNA pseudouridylation: new insights into an old modification. Trends Biochem. Sci. 38 (4), 210-218. doi: 10.1016/j.tibs.2013.01.002

Gevrey, M., Dimopoulos, I., and Lek, S. (2003). Review and comparison of methods to study the contribution of variables in artificial neural network models. Ecol. Model. 160 (3), 249-264. doi: 10.1016/S0304-3800(02)00257-0

Gulko, B., Hubisz, M. J., Gronau, I., and Siepel, A. (2015). A method for calculating probabilities of fitness consequences for point mutations across the human genome. Nat. Genet. 47 (3), 276-283. doi: 10.1038/ng.3196

Guzzi, N., Cieśla, M., Ngoc, P. C. T., Lang, S., Arora, S., Dimitriou, M., et al. (2018). Pseudouridylation of tRNA-derived fragments steers translational control in stem cells. Cell 173 (5), 1204-1216 e26. doi: 10.1016/j.cell.2018.03.008

He, J., Fang, T., Zhang, Z., Huang, B., Zhu, X., and Xiong, Y. (2018). PseUI: Pseudouridine sites identification based on RNA sequence information. $B M C$ Bioinf. 19 (1), 306. doi: 10.1186/s12859-018-2321-0

Jack, K., Bellodi, C., Landry, D. M., Niederer, R. O., Meskauskas, A., Musalgaonkar, S., et al. (2011). rRNA pseudouridylation defects affect ribosomal ligand binding and translational fidelity from yeast to human cells. Mol. Cell 44 (4), 660-666. doi: 10.1016/j.molcel.2011.09.017

Jacob, R., Zander, S., and Gutschner, T. (2017). The dark side of the epitranscriptome: chemical modifications in long non-coding RNAs. Int. J. Mol. Sci. 18 (11), 2387. doi: 10.3390/ijms18112387

Karijolich, J., and Yu, Y. T. (2011). Converting nonsense codons into sense codons by targeted pseudouridylation. Nature 474 (7351), 395-398.

Karijolich, J., Yi, C., and Yu, Y.-T. (2015). Transcriptome-wide dynamics of RNA pseudouridylation. Nat. Rev. Mol. Cell Biol. 16 (10), 581. doi: 10.1038/nrm4040

Ke, S., Pandya-Jones, A., Saito, Y., Fak, J. J., Vagbo, C. B., Geula, S., et al. (2017). $\mathrm{m}(6) \mathrm{A}$ mRNA modifications are deposited in nascent pre-mRNA and are not required for splicing but do specify cytoplasmic turnover. Genes Dev. 31 (10), 990-1006. doi: 10.1101/gad.301036.117

Khoddami, V., Yerra, A., Mosbruger, T. L., Fleming, A. M., Burrows, C. J., and Cairns, B. R. (2019). Transcriptome-wide profiling of multiple RNA modifications simultaneously at single-base resolution. Proc. Natl. Acad. Sci. U. S. A. 116 (14), 6784-6789. doi: 10.1073/pnas.1817334116

Kierzek, E., Malgowska, M., Lisowiec, J., Turner, D. H., Gdaniec, Z., and Kierzek, R. (2014). The contribution of pseudouridine to stabilities and structure of RNAs. Nucleic Acids Res. 42 (5), 3492-3501. doi: 10.1093/nar/gkt1330

Lawrence, M., Huber, W., Pages, H., Aboyoun, P., Carlson, M., Gentleman, R., et al. (2013). Software for computing and annotating genomic ranges. PloS Comput. Biol. 9 (8), e1003118. doi: 10.1371/journal.pcbi.1003118

Li, J.-H., Liu, S., Zhou, H., Qu, L.-H., and Yang, J.-H. (2013). starBase v2.0: decoding miRNA-ceRNA, miRNA-ncRNA and protein-RNA interaction networks from large-scale CLIP-Seq data. Nucleic Acids Res. 42 (D1), D92-D97.

Li, X., Zhu, P., Ma, S., Song, J., Bai, J., Sun, F., et al. (2015). Chemical pulldown reveals dynamic pseudouridylation of the mammalian transcriptome. Nat. Chem. Biol. 11 (8), 592-597. doi: 10.1038/nchembio.1836

Li, Y. H., Zhang, G., and Cui, Q. (2015). PPUS: a web server to predict PUSspecific pseudouridine sites. Bioinformatics 31 (20), 3362-3364. doi: 10.1093/ bioinformatics/btv366

Liu, H., Yue, D., Chen, Y., Gao, S. J., and Huang, Y. (2010). Improving performance of mammalian microRNA target prediction. BMC Bioinf. 11, 476. doi: 10.1186/1471-2105-11-476

Liu, K., Chen, W., and Lin, H. (2019). XG-PseU: an eXtreme Gradient Boosting based method for identifying pseudouridine sites. Mol. Genet. Genomics. 295, 13-21.

Liu, L., Lei, X., Meng, J., and Wei, Z. (2020). WITMSG: Large-scale prediction of human intronic m6A RNA methylation sites from sequence and genomic features. Curr. Genomics. 21, 67-76.

Lorenz, R., Bernhart, S. H., Zu Siederdissen, C. H., Tafer, H., Flamm, C., Stadler, P. F., et al. (2011). ViennaRNA Package 2.0. Algorithms Mol. Biol. 6 (1), 26.

Lovejoy, A. F., Riordan, D. P., and Brown, P. O. (2014). Transcriptome-wide mapping of pseudouridines: Pseudouridine synthases modify specific mRNAs in S. cerevisiae. PloS One 9 (10), e110799. doi: 10.1371/ journal.pone.0110799
McCleverty, C. J., Hornsby, M., Spraggon, G., and Kreusch, A. (2007). Crystal structure of human Pus10, a novel pseudouridine synthase. J. Mol. Biol. 373 (5), 1243-1254. doi: 10.1016/j.jmb.2007.08.053

Mei, Y. P., Liao, J. P., Shen, J., Yu, L., Liu, B. L., Liu, L., et al. (2012). Small nucleolar RNA 42 acts as an oncogene in lung tumorigenesis. Oncogene 31 (22), 27942804. doi: $10.1038 /$ onc. 2011.449

Meyer, K. D., and Jaffrey, S. R. (2017). Rethinking m(6)A readers, writers, and erasers. Annu. Rev. Cell Dev. Biol. 33, 319-342. doi: 10.1146/annurev-cellbio-100616-060758

Penzo, M., Guerrieri, A., Zacchini, F., Treré, D., and Montanaro, L. (2017). RNA Pseudouridylation in physiology and medicine: for better and for worse. Genes 8 (11), 301.

Safra, M., Nir, R., Farouq, D., Vainberg Slutskin, I., and Schwartz, S. (2017). TRUB1 is the predominant pseudouridine synthase acting on mammalian mRNA via a predictable and conserved code. Genome Res. 27 (3), 393-406. doi: 10.1101/gr.207613.116

Schwartz, S., Bernstein, D. A., Mumbach, M. R., Jovanovic, M., Herbst, R. H., Leon-Ricardo, B. X., et al. (2014). Transcriptome-wide mapping reveals widespread dynamic-regulated pseudouridylation of ncRNA and mRNA. Cell 159 (1), 148-162. doi: 10.1016/j.cell.2014.08.028

Shaheen, R., Han, L., Faqeih, E., Ewida, N., Alobeid, E., Phizicky, E. M., et al. (2016). A homozygous truncating mutation in PUS3 expands the role of tRNA modification in normal cognition. Hum. Genet. 135 (7), 707-713. doi: 10.1007/ s00439-016-1665-7

Shaheen, R., Tasak, M., Maddirevula, S., Abdel-Salam, G. M., Sayed, I. S., Alazami, A. M., et al. (2019). PUS7 mutations impair pseudouridylation in humans and cause intellectual disability and microcephaly. Hum. Genet. 138 (3), 231-239. doi: 10.1007/s00439-019-01980-3

Siepel, A., Bejerano, G., Pedersen, J. S., Hinrichs, A. S., Hou, M., Rosenbloom, K., et al. (2005). Evolutionarily conserved elements in vertebrate, insect, worm, and yeast genomes. Genome Res. 15 (8), 1034-1050. doi: 10.1101/ gr.3715005

Wang, K., Li, M., and Hakonarson, H. (2010). ANNOVAR: functional annotation of genetic variants from high-throughput sequencing data. Nucleic Acids Res. 38 (16), e164-e164. doi: 10.1093/nar/gkq603

Wong, Y.-H., Lee, T.-Y., Liang, H.-K., Huang, C.-M., Wang, T.-Y., Yang, Y.-H., et al. (2007). KinasePhos 2.0: a web server for identifying protein kinase-specific phosphorylation sites based on sequences and coupling patterns. Nucleic Acids Res. 35 (suppl_2), W588-W594.

Xuan, J.-J., Sun, W.-J., Lin, P.-H., Zhou, K.-R., Liu, S., Zheng, L.-L., et al. (2017). RMBase v2.0: deciphering the map of RNA modifications from epitranscriptome sequencing data. Nucleic Acids Res. 46 (D1), D327-D334.

Xue, H., Wei, Z., Chen, K., Tang, Y., Wu, X., Su, J., et al. (2020). Prediction of RNA methylation status from gene expression data using classification and regression methods. Evol. Bioinf.

Yang, H., Lv, H., Ding, H., Chen, W., and Lin, H. (2018). iRNA-2OM: a sequencebased predictor for identifying 2'-O-Methylation sites in homo sapiens. J. Comput. Biol. 25 (11), 1266-1277. doi: 10.1089/cmb.2018.0004

Zaringhalam, M., and Papavasiliou, F. N. (2016). Pseudouridylation meets next-generation sequencing. Methods 107, 63-72. doi: 10.1016/j.ymeth.2016.03.001

Zhao, X., Patton, J. R., Davis, S. L., Florence, B., Ames, S. J., and Spanjaard, R. A. (2004). Regulation of nuclear receptor activity by a pseudouridine synthase through posttranscriptional modification of steroid receptor RNA activator. Mol. Cell 15 (4), 549-558. doi: 10.1016/j.molcel. 2004.06.044

Zhu, Y., Xu, G., Yang, Y. T., Xu, Z., Chen, X., Shi, B., et al. (2018). POSTAR2: deciphering the post-transcriptional regulatory logics. Nucleic Acids Res. 47 (D1), D203-D211.

Conflict of Interest: The authors declare that the research was conducted in the absence of any commercial or financial relationships that could be construed as a potential conflict of interest.

Copyright ( 2020 Song, Tang, Wei, Liu, Su, Meng and Chen. This is an open-access article distributed under the terms of the Creative Commons Attribution License (CC $B Y)$. The use, distribution or reproduction in other forums is permitted, provided the original author(s) and the copyright owner(s) are credited and that the original publication in this journal is cited, in accordance with accepted academic practice. No use, distribution or reproduction is permitted which does not comply with these terms. 